\title{
Cooperative Control of UAV Rendezvous
}

Timothy W. McLain

Brigham Young University - Provo, mclain@byu.edu

Phillip R. Chandler

Air Force Research Laboratory

Steven Rasmussen

Veridian Inc., Air Force Research Laboratory

Meir Pachter

Dept. of Electrical Engineering, Air Force Institute of Technology

Follow this and additional works at: https://scholarsarchive.byu.edu/facpub

Part of the Electrical and Computer Engineering Commons, and the Mechanical Engineering

\section{Commons}

\section{Original Publication Citation}

McLain, T., Chandler, P., Rasmussen, S., and Pachter, M. Cooperative Control of UAV Rendezvous, Proceedings of the American Control Conference, vol. 3, pp. 2309-2314, June 2001, Arlington, Virginia.

\section{BYU ScholarsArchive Citation}

McLain, Timothy W.; Chandler, Phillip R.; Rasmussen, Steven; and Pachter, Meir, "Cooperative Control of UAV Rendezvous" (2001). Faculty Publications. 1534.

https://scholarsarchive.byu.edu/facpub/1534

This Peer-Reviewed Article is brought to you for free and open access by BYU ScholarsArchive. It has been accepted for inclusion in Faculty Publications by an authorized administrator of BYU ScholarsArchive. For more information, please contact ellen_amatangelo@byu.edu. 


\title{
Cooperative Control of UAV Rendezvous
}

\author{
Timothy W. McLain ${ }^{1 *} \quad$ Phillip R. Chandler ${ }^{2} \quad$ Steven Rasmussen $^{3} \quad$ Meir Pachter $^{4}$ \\ ${ }^{1}$ Department of Mechanical Engineering, Brigham Young University, Provo, Utah 84602 \\ ${ }^{2}$ Air Vehicles Directorate, Air Force Research Laboratory, WPAFB, Ohio 45433 \\ ${ }^{3}$ Veridian, Inc., Air Force Research Laboratory, WPAFB, Ohio 45433 \\ ${ }^{4}$ Department of Electrical Engineering, Air Force Institute of Technology, WPAFB, Ohio 45433
}

\begin{abstract}
The cooperative control of timing and synchronization of tasks of multiple unmanned air vehicles (UAVs) represents a valuable capability for a wide range of potential multi-UAV missions. This research addresses the specific problem of cooperative rendezvous in which multiple UAVs are to arrive at their targets simultaneously. The development of a rendezvous manager state machine and a cooperative control decomposition approach are described. Simulation results demonstrating the feasibility of the approach are presented.
\end{abstract}

\section{Introduction}

Recent advances in computation, sensors, and communication have provided the enabling technologies for achieving cooperative control of multiple-vehicle systems. To make cooperation among multi-vehicle teams viable, the development of cooperative control strategies is required. This paper focuses on the development and application of a technique for coordinating the timing of activities among a team of unmanned air vehicles. For UAVs, the ability to synchronize activities provides an important strategic capability in a wide variety of military missions.

Research in the cooperative control of UAVs has been limited. Much of the work reported in the literature focuses on close formation flight of multiple-vehicle systems. The dynamics of these formations are tightly coupled either by control laws governing the formation behavior [1] or by a combination of aerodynamics and formation control laws $[6,9]$. Because these systems are coupled dynamically, they can analyzed as a single, largescale system.

More relevant to this work is the control of spatially distributed UAV systems that are not coupled dynamically by aerodynamic forces or low-level control laws. The decomposition method presented here was first applied to a simple UAV rendezvous problem in [5]. UAV rendezvous was also treated in [2], where focus of the work was the development of a near-real-time planner that generated flyable paths. In [8] a cooperative target classification strategy for a system of low-cost autonomous attack vehicles is presented.

\footnotetext{
*Corresponding author, email: tmclain@et.byu.edu
}

\section{Problem Statement}

To aid in the development of a cooperative control strategy, a benchmark rendezvous problem was formulated. A schematic representation of this benchmark problem scenario is shown in Figure 1.

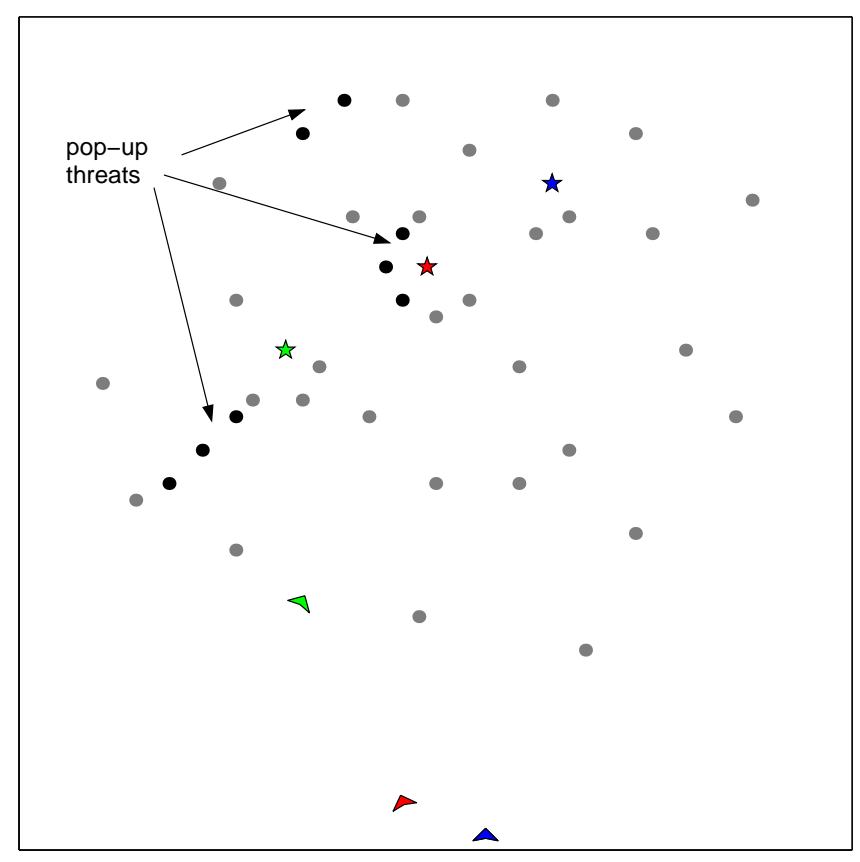

Figure 1: Rendezvous benchmark problem

In this scenario, there are three UAVs, each having an assigned target. Threats (e.g., enemy radar) are of two types: known and pop-up. The locations of known threats are known by the UAVs at the start of the mission. The locations of pop-up threats are only known by the UAVs when they come within range of one of the UAV's threat sensors. The objective is for the UAVs to arrive at their targets simultaneously with sufficient fuel to return to base while minimizing the risk of detection by threats. From a strategic standpoint, simultaneous arrival at the targets increases the element of suprise and decreases the risk of detection. Once the targets have been hit, the cooperative control must plan the egress of the UAVs.

Under certain conditions, a UAV may be required to drop out of the mission. For example, if a UAV's fuel availability approaches the amount required to return to 
base, it should drop out of the rendezvous mission and return. The increased risk imposed by pop-up threats may also result in the need to reduce the number of UAVs participating in the rendezvous. The rendezvous manager cooperative control strategy presented here effectively handles these mission situations.

\section{Technical Approach}

\subsection{Rendezvous Manager Statechart}

In this research, simulation of UAV rendezvous has been implemented in the Matlab/Simulink programming environment. Specifically, the rendezvous manager has been implemented as a statechart in the Stateflow graphical design tool. The control of each UAV participating in the mission is managed by the rendezvous manager statechart (RMS), which is depicted in Figure 2. The RMS runs concurrently on each UAV participating in the rendezvous. In the statechart, there are three main states: Plan Rendezvous, Plan Egress, and Execute Plan. Transitions from one state to another occur when the transition statements (shown in brackets) become true. For example, if a UAV is in the Execute Plan state and its fuel level drops below a predetermined limit, then the RMS transitions to the Plan Egress state. When the planning of egress is complete the RMS transitions back to the Execute Plan state where the egress trajectory is processed to provide desired heading and desired velocity commands to the UAV.

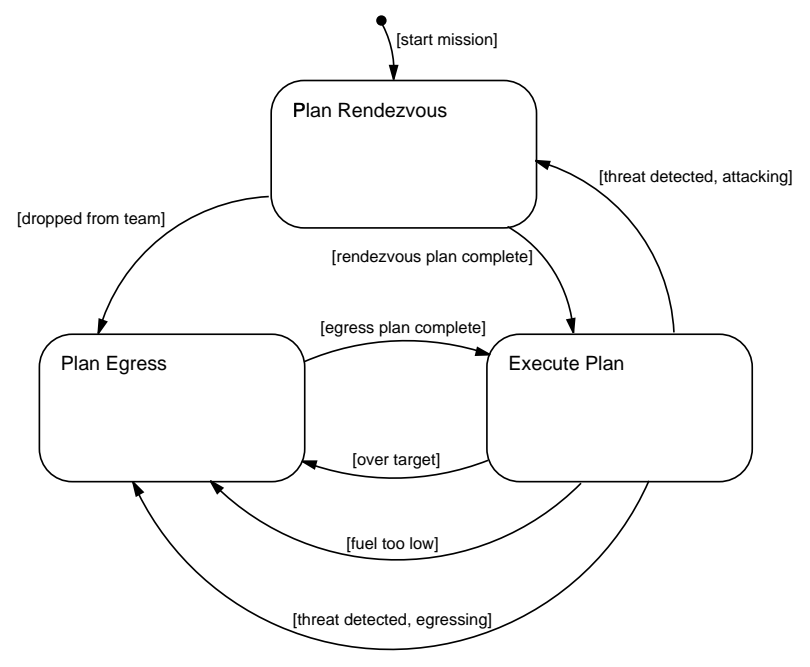

Figure 2: Rendezvous manager statechart

Upon transitioning between the main states of the mission, various Matlab functions are called that control the manner in which the rendezvous mission is carried out. Upon entering the Plan Rendezvous state, the calculations for coordinating rendezvous are done. This includes calculating candidate paths through the threats, calculating and communicating possible ETA ranges, calculating feasible team ETA ranges, calculating and communicating coordination functions, calculation of the optimal team ETA, and selection of the best path to achieve the team ETA. Within the Plan Rendezvous state, interactions with a resource allocation manager can be modeled. The resource allocation manager determines how many UAVs should compose the team. In its current form, the rendezvous manager starts with three UAVs and can successively drop UAVs from the team so that it is composed of two, one, or zero UAVs.

In the Plan Egress state, a path to home base or another egress point is calculated. The egress point can be determined based the state being transitioned from. For example, if a UAV is low on fuel, the egress point might be home base, whereas if the UAV has completed the mission, the egress point might be a preplanned location where it would join formation with the other UAV team members.

In the Execute Plan state, the path description generated by the path planner (either in the Plan Rendezvous state or the Plan Egress state) is processed in time to produce the desired heading command for the UAV. The desired velocity command is specified by the planner. Also in the Execute Plan state, a radar function scans the battle area for pop-up threats and calculates the range to the target. Fuel level is also monitored here.

Using this relatively simple statechart, a fairly complicated set of operational situations can be dealt with effectively. Changes in the threat scenario, team composition, fuel status, and other operational conditions are handled without significant difficulty.

\subsection{Path Planning}

Planning the rendezvous of multiple UAVs requires a two-dimensional path planning algorithm suitable for UAVs. For a scenario such as the benchmark problem of Figure 1, the path planner for each UAV must produce a family of paths from its initial location to its target. The cost for each path in the family of paths is based on the proximity of the path to the threats. To be suitable, the planner must produce paths that avoid the threats and are flyable by the UAVs. This flyability constraint can be handled by ensuring that the curvature of the paths produced is sufficiently small.

In this work, the path planning approach described in [4] was used. This planner has a number of useful features, including the ability to lengthen the path, specify path length, and smooth the path to make it flyable and move away from threats. Other planners, such as that described in [2], could be used provided that they yield flyable paths that avoid threats. To clarify the discussion of the decomposition strategy, a brief description of the planning approach is given.

As the main objective in generating a path to the target is to avoid threats, a logical approach for generating an initial path to the target is to construct a Voronoi diagram based on the locations of the threats. Figure 3 shows a Voronoi diagram for a particular threat configuration and the resulting lowest-cost flyable path produced by the planner. Taken in different combinations, the edges of the Voronoi diagram provides a rich set of 
paths from the starting point to the target.

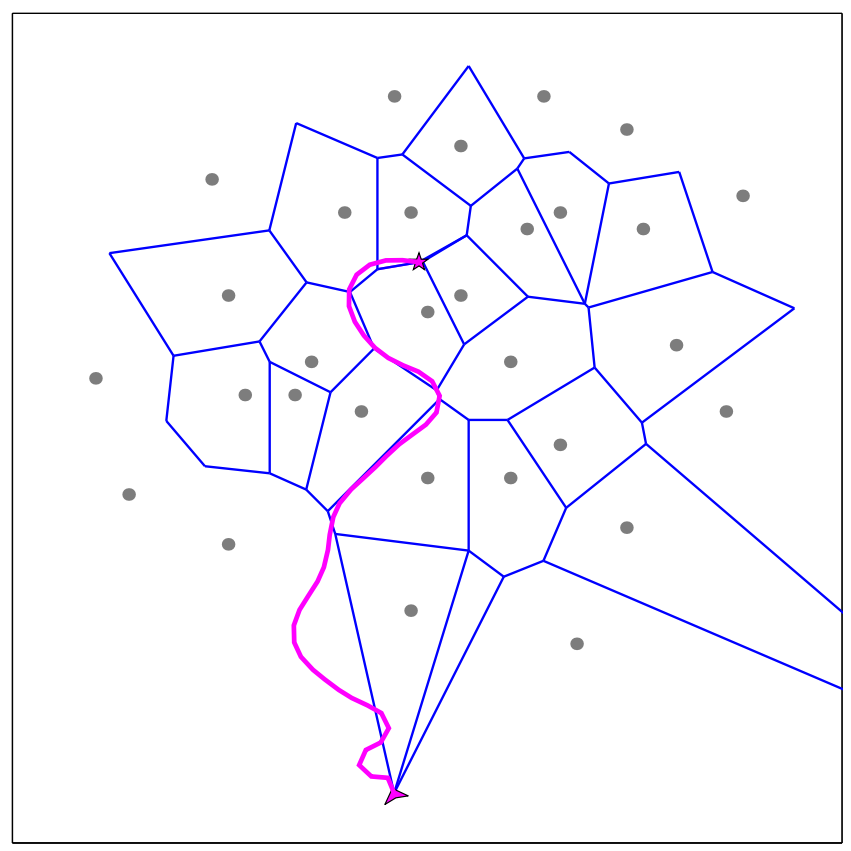

Figure 3: Voronoi diagram and flyable path

The $k$ lowest-cost paths through the diagram to the target are determined using a variation of Eppstein's $k$ shortest paths algorithm [3]. The cost for traversing an edge of the Voronoi diagram is proportional to the accumulated energy of the UAV's radar signature as it travels along the edge. The strength of the signature is proportional to the inverse of the distance to the threat to the fourth power. All of the threats are considered when calculating the cost.

The final step in the path-planning process is the discretization and smoothing of the candidate paths to the target. For the results presented here, the paths have been discretized into $1 \mathrm{~km}$ segments and then smoothed to give flyable paths that avoid the threats. An example of a discretized and smoothed path is shown in Figure 3. The s-turn at the beginning of the path demonstrates the path-lengthening feature of the planner that was used. Further details of this planner can be found in [4].

The result of the discretization and smoothing processes is a path description for the UAV that is expressed in terms of the $x$ and $y$ locations of the path waypoints

$$
\xi \triangleq\left(\begin{array}{ccccc}
x_{0} & x_{1} & x_{2} & & x_{n} \\
y_{0} & y_{1} & y_{2} & \ldots & y_{n}
\end{array}\right)
$$

where $n-1$ is the number of intermediate waypoints, $\left(x_{0}, y_{0}\right)$ denotes the initial point, and $\left(x_{n}, y_{n}\right)$ denotes the target point. In the subsequent discussion, the notation $\xi_{i, j}$ will be used to describe the $j^{t h}$ path for the $i^{t h}$ vehicle. The concept of the UAV trajectory differs only slightly from that of the UAV path. The path describes where the UAV goes. The trajectory describes where the UAV goes as a function of time. If the UAVs fly with constant velocity, then the trajectory of the $i^{\text {th }} \mathrm{UAV}$ is parameterized by its path description $\xi_{i}$ and its velocity along the path $v_{i}$.

\subsection{Decomposition Strategy}

In the Plan Rendezvous state of Figure 2, the calculations that enable the cooperative control of rendezvous are performed. By building on the decomposition strategy originally proposed in [5], rendezvous of multiple vehicles can be performed in a decentralized, yet teamoptimal manner. The decomposition strategy is based on selection of coordination variables and the determination of coordination functions to be shared with other UAVs composing team.

The objective of the rendezvous problem considered here is for the UAVs composing the team to arrive at their targets simultaneously. En route to their targets, the UAVs should avoid threats and conserve a sufficient amount of fuel to return to their home base. For each $\mathrm{UAV}$, numerous candidate paths to the target are found by the path planner. The procedure for selecting which path to follow will now be described.

Trajectories during the mission are calculated to maximize the survivability of the team, while ensuring that the rendezvous at the target occurs as desired. The survivability of the vehicles is quantified by a single cost function representing the threat cost $J_{t, i}$ to each vehicle. This cost, as well as fuel consumption, is directly affected by the UAV trajectory. Fuel consumption is a function of UAV speed and path length. Threat cost is a function of time spent in high threat regions, which also depends on UAV speed and path length.

The task of finding the best way to coordinate rendezvous can be posed as an optimization problem. The problem becomes one of choosing $\xi_{i}$ and $v_{i}, i=1,2, \ldots, N$, that minimize the cost

$$
J=\sum_{i=1}^{N} J_{t, i}\left(\xi_{i}, v_{i}\right),
$$

for the $N$ vehicles, while constraining them to arrive at the target simultaneously. For the benchmark problem, this optimization must be carried out prior to entering the battle area and when changes in the threat scenario are detected. This optimization could be performed in a centralized manner by having all state, threat, and fuel information for each UAV communicated to a central location where a large-scale optimization problem would be solved and trajectory information communicated back to individual UAVs. A preferred alternative is to decentralize the computational solution of the optimization problem by allowing each UAV to compute its own trajectory that is optimal with respect to the needs of the team. The challenge here is to determine what information must be communicated among team members to give them an awareness of the situation of the other team members so that each may calculate solutions that are optimal from a team perspective.

The decomposition strategy utilized here allows these 
optimization computations to be carried out in a decentralized manner. The proposed decomposition method has similarities to decomposition strategies developed for multidisciplinary optimization purposes [7]. For the coordinated control problem, the purpose of decomposition is to break up a single, very large optimization problem into smaller, more tractable optimization problems that will allow computations to be decentralized among the UAVs, that will require only modest communication among UAVs, while taking into account the threat and fuel situation of each UAV.

Figure 4 shows the decomposition method developed to for rendezvous. To achieve rendezvous, an estimated time until arrival (ETA or $t_{a}$ for brevity) for the team is chosen that each UAV can meet. The team objective is to choose an ETA that allows the UAVs to follow trajectories that minimize the collective threat exposure of the team. In this problem, ETA is termed a coordination variable since its selection and imposition on the individual UAVs results in their simultaneous arrival at their respective targets.

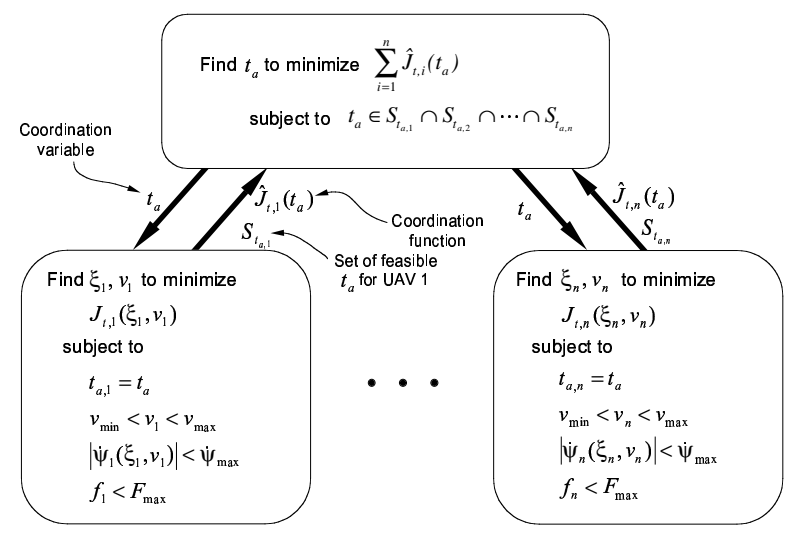

Figure 4: Decomposition strategy for cooperative rendezvous

At the level of the individual UAV, the objective is to plan a trajectory that will allow the desired team ETA to be met while avoiding threats. The trajectory must satisfy constraints on the UAV's velocity $\left(v_{i}\right)$, heading rate $\left(\dot{\psi}_{i}\right)$, and fuel $\left(f_{i}\right)$.

The trajectory planning elements of the mission are event driven and occur upon entry into the battle area and upon the detection of a previously unknown threat. When these events occur, each UAV calculates a function describing its own threat costs versus ETA. This function is termed a coordination function since it describes how changes in the team ETA (coordination variable) affect the threat cost of an individual UAV.

Figure 5 depicts the coordination function information for three UAVs. This information is based on the discretized paths returned from the $k$ best paths search of the Voronoi graph. Each path through the Voronoi graph results in a line segment on the coordination function plot. While each path has a fixed length, each possible velocity along the path will result in a distinct ETA and threat cost. As velocity decreases, both ETA and threat cost increase. However, paths that are longer tend to do a better job of avoiding threats. The result is that the individual coordination function segments increase with ETA, while for a group of paths, the coordination function tends to decrease with increasing ETA.

The coordination functions for the UAVs composing the team are passed to the team level where they are used to calculate an optimal ETA for the team. This optimal team ETA is then passed back to the vehicle level where it is used to determine new trajectories for each of the vehicles. Figure 5 represents the determination of the team-optimal ETA graphically. Threat cost information for three UAVs is shown. Each UAV has a set of ETAs that it can achieve. The team-optimal ETA must lie on the intersection of these sets (depicted by bold lines). The team-optimal ETA is that ETA which minimizes the sum of the threat costs for each of the UAVs. For the coordination functions of Figure 5, an ETA of $325 \mathrm{sec}$ minimizes the cumulative threat cost of the team.

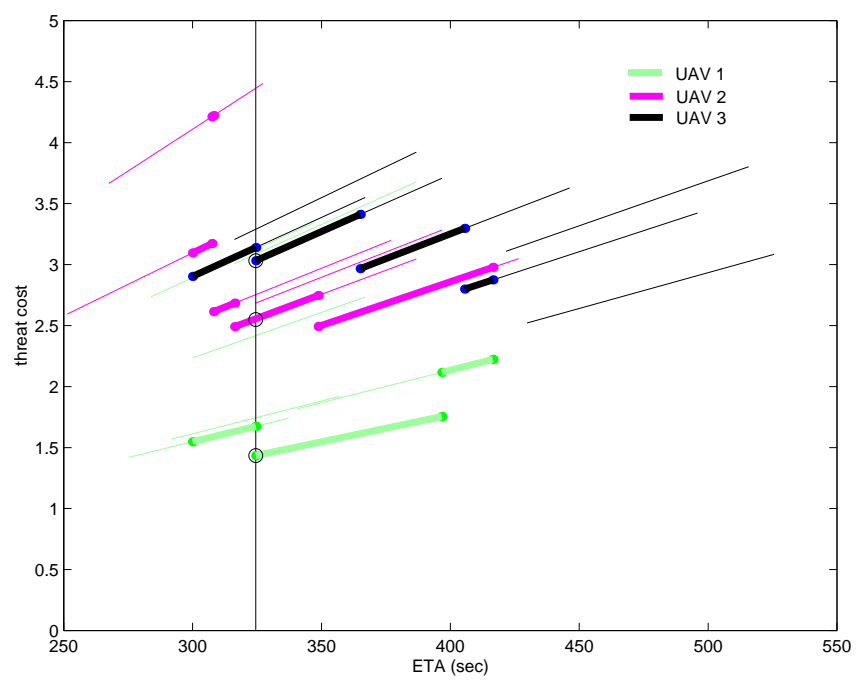

Figure 5: Optimal ETA determination

It should be noted that coordination function information is only calculated for regions where the ETAs for the three UAVs overlap. In this way, a minimal amount of information is communicated. Furthermore, with piecewise linearly increasing coordination functions (as occur here), the optimal ETA will always occur at the lower bound of one the ETA overlap regions. Because of this, the ETA optimization can be carried out by exhaustive search with little computational effort.

The problem of optimizing trajectories for all the UAVs of a team involves searching over an infinite number of solutions (given that $x, y$, and $v$ can take on any value in their feasible range). In the approach presented, the search space of the optimization has been reduced dramatically by considering only finite number of paths (one for each Voronoi candidate path) and by assuming that velocity is constant along the path. 
Notice that the coordination function $\left(\hat{J}_{t, i}\right)$ is dependent on a single coordination variable $\left(t_{a}\right)$, while the threat cost $\left(J_{t, i}\right)$ is a function of the many parameters describing the trajectory $\left(\xi_{i}, v_{i}\right)$. This reduction in the dimensionality enabled by the use of the coordination function is a key feature of the decomposition strategy.

Once an optimal ETA is determined, it is passed to the vehicle level where the best trajectory (parameterized by $\xi_{i}$ and $v_{i}$ ) corresponding to the chosen ETA is calculated. Selection of $\xi_{i}$ and $v_{i}$ to give the desired ETA ensures the simultaneous arrival of the UAVs at the target.

\subsection{Resource Allocation}

During the course of the mission, it may be desirable to change the composition of the rendezvous team. For example, if threats pop up in an undesirable way, it may be best to drop one or more UAVs off of the team to reduce their risk of being detected. In some instances, the potential benefit of hitting a target may not be worth the risk associated with attacking it. The problem of determining the number of UAVs to participate in the rendezvous would belong to a resource allocation manager.

In this work, a resource allocation manager (RAM) with minimal functionality has been implemented. The input to the RAM is the minimum threat cost for the various numbers of team members possible. In this case, the minimum threat cost for a team of one UAV, for a team of two UAVs, and for a team of three UAVs is communicated to the RAM whenever there is a change in the threat scenario. Based on the acceptable level of risk, the RAM selects the number of UAVs to participate in the rendezvous $(0,1,2$, or 3$)$ and returns this number to the rendezvous manager. The rendezvous manager then selects which UAVs are to participate. Those UAVs dropped from the team transition to the Plan Egress state of the RSM and plan their way back to their home base.

\section{Results}

To demonstrate the utility of the rendezvous manager, simulation results are presented for a three-UAV team. The conditions of the mission, such as pop-up threat positions and UAV initial positions, have been chosen to exercise the full capability of the rendezvous manager.

Two different simulations are presented. In both simulations, the threat locations (both known and pop-up) and the UAV initial positions are the same. The two simulations are different in the way the resource allocation manager directs the mission. In the first simulation, the threat risk threshold in the RAM is set high enough so that all of the UAVs proceed to their targets. In the second simulation, the threat risk threshold is set so that one UAV will be dropped during the mission.

\section{Simulation 1: All UAVs Participating}

Figures 6 and 7 present the results for a mission where the acceptable risk level is sufficiently high to cause all
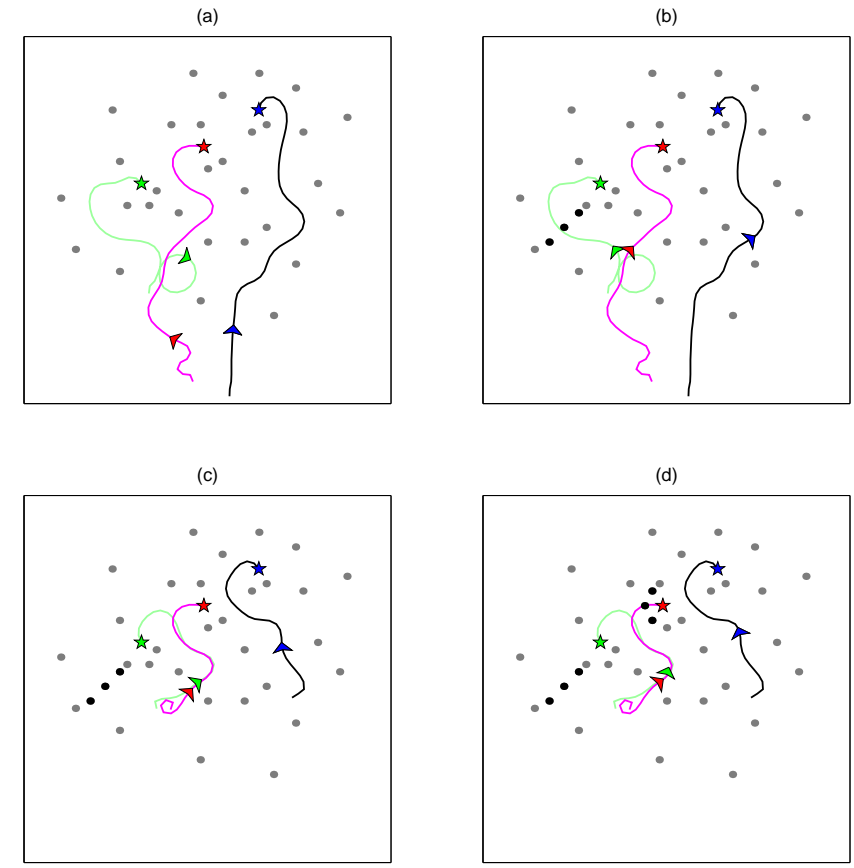

Figure 6: Simulation 1 results (part 1)

UAVs to participate in the rendezvous. Frame (a) of Figure 6 shows the three UAVs flying their initial paths. Of interest is the large loop in the light grey path and the s-curve at the beginning of the medium grey path. It was necessary to add length to these paths initially to achieve an overlap in the UAV ranges so that rendezvous could occur.
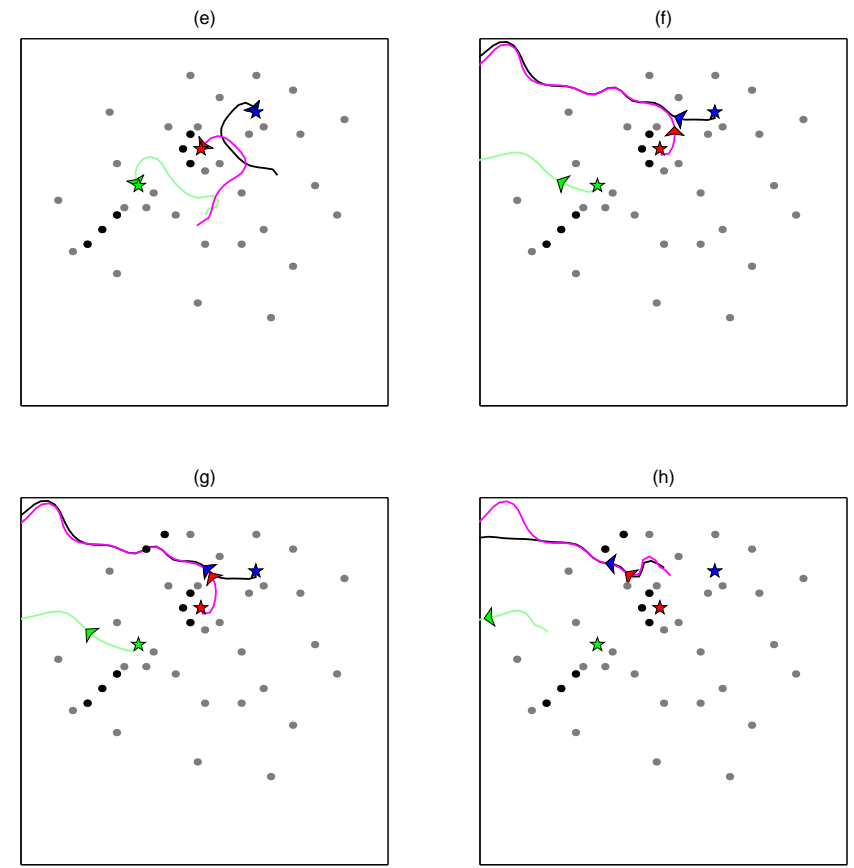

Figure 7: Simulation 1 results (part 2)

Frame (b) depicts the detection of the first set of pop- 
up threats, which are shown by the black circles. Upon detection of these threats, new trajectories to the target are planned. Frame (c) shows the UAVs flying the new trajectories. Frame (d) shows the detection of a second set of pop-up threats. Once again, trajectories to the targets are replanned. Frame (e) of Figure 7 shows the UAVs flying the newest trajectories and arriving at their targets simultaneously. Notice how the paths to the targets have changed to avoid the pop-up threats. It should be noted that the trajectories selected not only enable simultaneous arrival at the targets, but they also represent the lowest-threat-cost paths to the target from a team perspective.

The final phase of the mission is egress which is represented in frames (f), (g), and (h) of Figure 7. In frame (f), the initial egress paths are shown. Frame (g) shows a pop-up threat being detected during egress and frame $(h)$ shows the final paths out of the battle area.

\section{Simulation 2: One UAV Dropped}

The simple resource allocation manager that has been included in the simulation model allows a threat risk threshold to be specified. During the course of a mission, the RAM may determine that it would be too risky to have all three UAVs attack their targets, and specify that one or more UAVs be dropped from the rendezvous team.
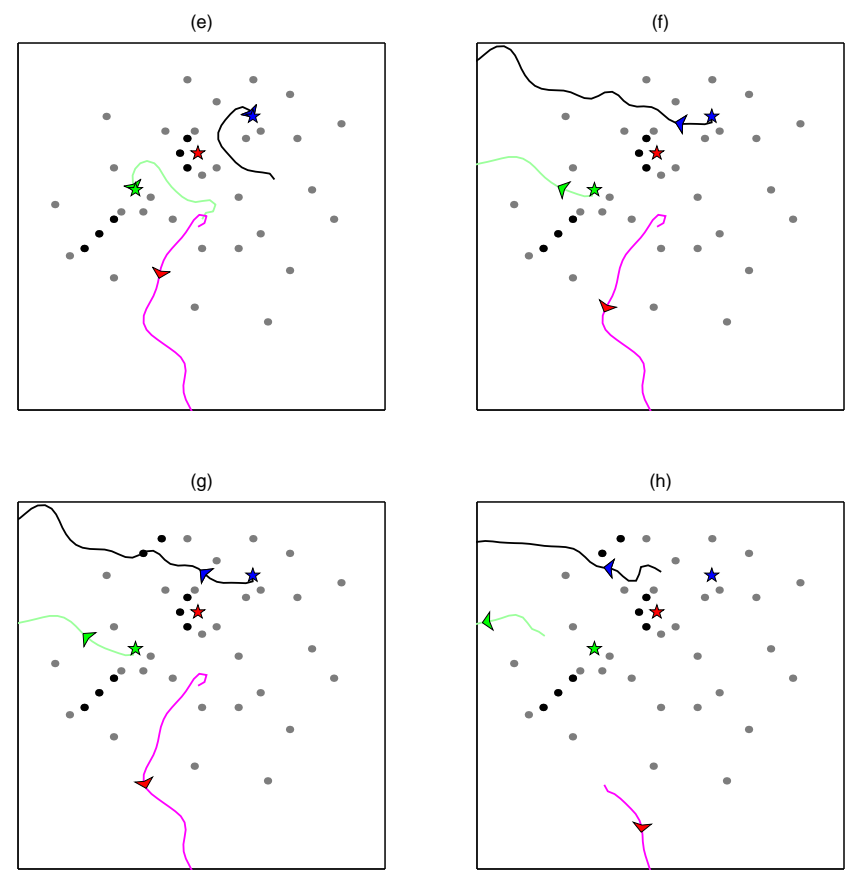

Figure 8: Simulation 2 results (part 2)

In this simulation, the threat risk threshold is lowered to the point that one UAV is dropped from the team. For the first four frames of the mission, Simulation 2 results are identical to those for Simulation 1 shown in Figure 6. Upon detecting the first pop-up threats (frames (b) and (c)), the UAV team can replan in a way that the threat threshold is not exceeded. However, upon detecting the second set of pop-up threats (frame (d)), the threat threshold is exceeded. The result is that the medium gray UAV is dropped from the team. This can be seen in frame (e) of Figure 8 where the medium gray UAV is shown flying to its home base. Frame (e) shows the light gray and black UAVs arriving at their targets simultaneously.

Frame (f) of Figure 8 shows the medium gray UAV en route to its home base, while the light gray and black UAVs are en route to their egress locations. Frame (g) shows the detection of a pop-up threat during egress. In frame (h), the change in paths for all of the UAVs is shown. Upon detection of a threat by any UAV, all UAVs replan their paths even though the threats may not be nearby.

\section{Conclusions}

The research presented here has addressed the cooperative control of rendezvous of multiple UAVs at predetermined target locations. As an approach for finding a solution that is best for the team as a whole, a decomposition strategy was developed that allows teamoptimal solutions to be computed in a decentralized manner. Simulation results have demonstrated the capabilities of the rendezvous manager statechart, the resource allocation manager, and the cooperative control decomposition strategy developed for this work.

\section{References}

[1] M. R. Anderson And A. C. RobBins, Formation flight as a cooperative game, in Proc. of the AIAA GN\&C Conf., 1998, pp. 244-251.

[2] P. Chandler, S. Rasmussen, and M. Pachter, UAV cooperative path planning, in Proc. of the AIAA GN\&C Conf., August 2000.

[3] D. EPPSTEIN, Finding the $k$ shortest paths, SIAM Journal of Computing, 28 (1999), pp. 652-673.

[4] T. MCLAIN AND R. BEARD, Trajectory planning for coordinated rendezvous of unmanned air vehicles, in Proc. of the AIAA GN\&C Conf., August 2000.

[5] T. Mclain, P. Chandler, and M. Pachter, A decomposition strategy for optimal coordination of unmanned air vehicles, in Proc. of the ACC, June 2000.

[6] A. Proud, M. Pachter, And J. D'Azzo, Close formation control, in Proc. of the AIAA GN\&C Conf., August 1999.

[7] J. Sobieszczanski-Sobieski, J. Barthelemy, And G. GILES, Aerospace engineering design by systematic decomposition, Proc. of the AIAA, 2 (1984).

[8] D. Swaroop, A method of cooperative classification for LOCAAS vehicles, tech. rep., Air Force Research Laboratory, Air Vehicles Directorate, August 2000.

[9] J. D. Wolfe, D. F. Chichka, And J. L. Speyer, Decentralized controllers for unmanned aerial vehicle formation flight, in Proc. of the AIAA GN\&C Conf., 1996. 